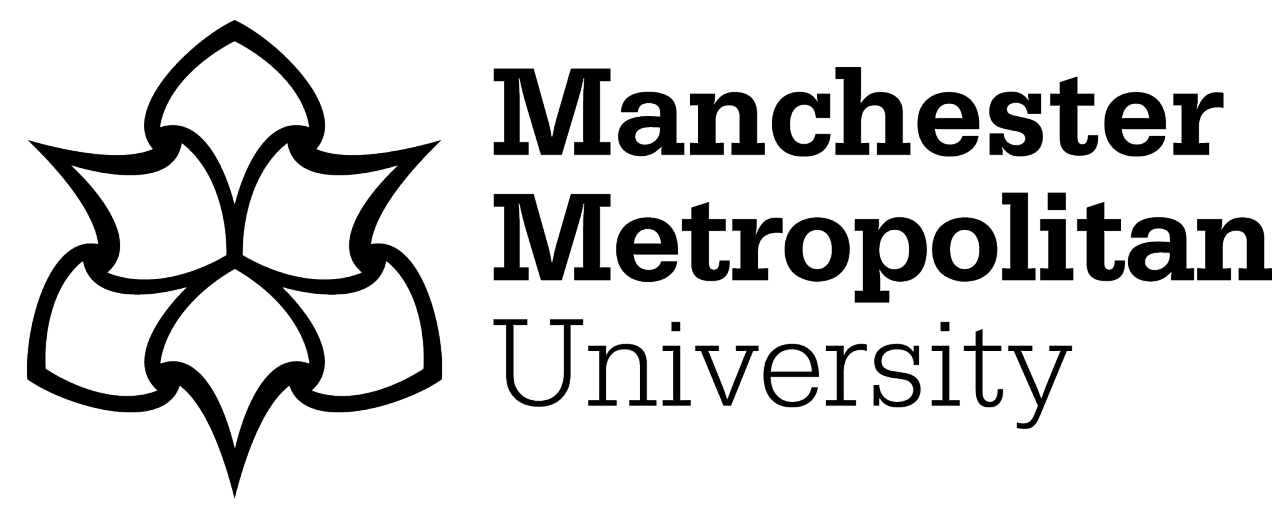

Parker, A and Dagnall, N (2018) Reduced Impact of Imagery Processing on False Autobiographical Recollection: The Effects of Dynamic Visual Noise. Memory, 27 (2). pp. 163-173. ISSN 0965-8211

Downloaded from: https://e-space.mmu.ac.uk/620949/

Version: Accepted Version

Publisher: Taylor \& Francis (Routledge)

DOI: https://doi.org/10.1080/09658211.2018.1495236

Please cite the published version 
Running Head: VISUAL IMAGERY, AUTOBIOGRAPHICAL MEMORY \& DYNAMIC VISUAL NOISE

\title{
Reduced Impact of Imagery Processing on False Autobiographical Recollection: The Effects of Dynamic Visual Noise.
}

\author{
Andrew Parker* \& Neil Dagnall \\ Manchester Metropolitan University \\ Department of Psychology \\ Brooks Building \\ 53 Bonsall Street \\ Manchester \\ M15 6GX
}

United Kingdom

* To whom correspondence should be addressed

e-mail: a.parker@mmu.ac.uk

Note: This is the final version of the authors copy. Subtle difference may exist between this and the final published version in Memory.

Telephone: 01612472586

Acknowledgements

The authors would like to thank Dr Steve Jachim for the construction of the Dynamic Visual Noise software used in this research. In addition, we would like to thank Kath Walmsley, Lara Shelley, Nicola Diggle, Rachel Russell and Lisha Dahyabhai for assistance with the collection of data for the experiments reported here. 


\begin{abstract}
Research has demonstrated that merely imagining an autobiographical event can bring about false memories for that event. One explanation for this is that imagination leads to the creation and incorporation of visual-imagistic information into the event representation. This idea was tested in two experiments in which visual-imagery processing was disrupted by the use of Dynamic Visual Noise (DVN). In Experiment 1, autobiographical memories that were rated as 'known' and lacking in event detail were subsequently rated as more 'remembered' following imagination. In Experiment 2, imagination led to improbable autobiographical events being rated as more believable and vivid. In both experiments, interfering with imagery processing by DVN reduced these effects. It was concluded that visual-imagistic processing plays an important role in altering the mnemonic status of autobiographical representations.
\end{abstract}

\title{
$\underline{\text { Keywords }}$
}

Imagination inflation

Autobiographical memory

Dynamic visual noise

False memory

Episodic memory 
Reducing the Impact of Imagery Processing on False Autobiographical Recollection: The Effects of Dynamic Visual Noise.

False memory defines that class of memories pertaining to cognitive representations that are mistakenly considered to have arisen as a result of a real experience (Schacter, 1999). Such memories can arise as a result of a number of processes that occur both inside and outside the psychological laboratory (Loftus \& Davis, 2006; Roediger \& McDermott, 1995). Autobiographical memory refers to personal memory about the self and comprises event-specific and more generalised knowledge and beliefs (Conway, 2005; Conway, \& Pleydell-Pearce, 2000; Levine, 2004). Accordingly, false autobiographical memories are recollections about events that were not in actual fact experienced. The experiments reported here are concerned with the effects of imagination on false autobiographical memory. In particular, the role of imagination and the use of dynamic visual noise as a technique to gauge the impact of visual imagery processes on recollection and believability of constructed events.

\section{False autobiographical memory}

Recent research has shown that false personal memories occur in real life (e.g., Crombag, Wagenaar, \& van Koppen, 1996; Loftus \& Davis, 2006; Odegard \& Lampinen, 2004), and can also be created in controlled contexts that allow their qualities and determination to be studied. One particularly well studied technique involves investigating the effects on memory of imagining performing non-performed actions or experiencing fictitious or unlikely events. For example, Goff and Roediger (1998) asked participants to perform a set of simple actions (e.g., unlocking a padlock). Subsequently, they were asked to merely imagine performing other actions 
that were not performed earlier. In a final memory test, it was revealed that the act of imagination created false memories for the non-performed actions.

These imagination inflation effects can also be found for experiences of a more personal and autobiographical nature. For instance, Garry, Manning, Loftus and Sherman (1996) asked participants to rate their certainty that an event from the past had actually occurred to them (e.g., winning a stuffed animal at a carnival). For some of these events, they were later guided through an imagination phase in which the experimenter led them to picture the events in their minds. Finally, they were again asked to rate their certainty that the events had actually occurred to them. Once more, it was found that imagination increased the belief in the actuality of the events occurring. These effects have been found to be robust and occur across a range of experiments varying in stimuli and aspects of procedure (e.g., Brewin \& Andrews, 2016; Frenda, Knowles, Saletan, \& Loftus, 2013; Lindner \& Echterhoff, 2015; Marsh, Pezdek, \& Lam, 2014; Thomas \& Loftus, 2002).

\section{Explaining false autobiographical memory}

False autobiographical memories can be explained as resulting from memory misattributions within the context of the Source Monitoring Framework (SMF) (Johnson, Hashtroudi \& Lindsay, 1993). According to this framework, mental experiences are attributed to particular sources (e.g., a past experienced occurrence) based on the appraisal of the qualities of the mental contents of the experience, the context in which the contents are embedded, and how together these are evaluated against particular decision criteria, goals and heuristics.

It has been found that the processes that lead to accurate memory attributions can also produce false memories. This is especially the case when the mental representation of real and false events share similar characteristics and inappropriate 
criteria and heuristics are employed to evaluate recalled details (e.g., Allen \& Lindsay, 1998; Lindsay, Johnson, \& Kwon, 1991; Lyle \& Johnson, 2007). In such instances, a mental representation might be incorrectly attributed to the past with the result being a false autobiographical memory. For example, real (vs. false) memories typically have associated with them more perceptual and sensory details (Johnson, Foley, Suengas, \& Raye, 1988; Mather, Henkel, \& Johnson, 1997; Okado, \& Stark, 2003). However, these qualities can also form part of non-experienced events if cognitive activities such as visualising or imagining have played a role in the creation or modification of that representation. Consequently, such a representation would be a prime candidate for misattribution to a past occurrence rather than imagination.

It is contended that imagination inflation effects are most likely when sensoryimagistic records are created during imagination itself; a contention that receives support from a number of experiments. For example, it has been found that imagination effects are typically greater in participants with higher visual imagery abilities (Horselenberg, Merckelbach, Muris, Rassin, Sijsenaar \& Spaan, 2000; Johnson, Raye, Wang \& Taylor 1979). In addition, imagery instructions that guide or prompt the use of sensory information, enhance imagination inflation effects (Bays, Zabrucky, \& Foley, 2015), and can lead to detailed (but false) recollection and reduced source discriminability (Thomas, Bulevich, \& Loftus, 2003). Finally, the visual perspective from which one imagines an event can also influence the magnitude of the inflation effect (Linder \& Echterhoff, 2015; Marsh, et al., 2014).

Further support for the assertion that imagination effects are related to sensory processing, is work in neuroimaging that has revealed the neural regions associated with such processing are more active on imagination trials that later produce false 
memories for imagined items attributed to actual presentation (Gonsalves, et al., 2004; Sugimori, Mitchell, Raye, Greene, \& Johnson, 2014).

Overall, imagination inflation effects can be explained by the SMF in which (i) sensory-perceptual attributes are generated and incorporated into the imagined acts and, (ii) subsequent memory for real (vs. imagined acts) reactivates those sensory attributes and individuals monitor for the presence of such information in order to make appropriate memory attributions. Although the background research outlined above is consistent with this idea, additional evidence for the importance of sensoryimagistic information, (in the case of the current experiment, visual-imagistic information), would be the finding that the use of a visual interference task disrupts inflation effects.

\section{Dynamic visual noise: Interfering with visual-imagistic processing}

One task shown to be valuable in examining the extent to which visualimagistic information is used to perform various cognitive activities is the dynamic visual noise (DVN) task (Quinn \& McConnell, 1996). The task involves viewing the display of a randomly changing array of small black and white squares on a computer screen. It has been previously established that DVN disrupts selectively the processing of visual-imagistic information. For example, Quinn and McConnell (1996), found that DVN reduced episodic memory for a word-list with instructions to use a visual mnemonic, but had no effect when instructed to use a non-visual strategy.

Subsequent work has extended and refined these findings by demonstrating that DVN effects can influence encoding or retrieval (Quinn and McConnell, 2006), recognition confidence (Kemps \& Andrade, 2012), the free recall and recognition of concrete (vs. abstract) words (Parker \& Dagnall, 2009), the specificity of autobiographical memories (Anderson, Dewhurst, \& Dean, 2017), and processing in 
non-memory tasks that require the use of mental imagery (Dean, Dewhurst, Morris, \& Whittaker, 2005).

These findings are explained by the influence of DVN on a component of visual short-term memory called the passive visual store (Quinn \& McConnell, 1996) or visual buffer (Kosslyn, Thompson, \& Ganis, 2006; Quinn \& McConnell, 2006). The function of the buffer is to hold and maintain visual and imagery-based information in short-term storage. The DVN field has obligatory access to this buffer and consequently interferes with the processing of visual representations that are currently active (Quinn \& McConnell, 2006).

In summary, the DVN task can be considered a good technique for assessing the effects of interfering selectively with visual-imagistic processing on performance on other tasks that are hypothesised to involve the use of visual-imagery. Accordingly, the DVN task should be able to provide a means to examine the contribution of visual-imagery processes to the effects of imagination on autobiographical memory.

\section{Experiment 1. Imagination \& DVN effects on 'remember' and 'know' judgements in autobiographical memory.}

Experiment 1 examined the contribution of imagery-based processes to 'remember' and 'know' judgements in autobiographical memory (Hyman, Gilstrap, Decker, \& Wilkinson, 1998). In the Hyman et al. experiments, participants first generated a number of 'remembered' and 'known' autobiographical memories. A 'remember' type memory was defined as one that involved conscious awareness of an event or experience that included the presence of much associated details like visual images, and the feeling that the memory is veridical. A 'know' memory was defined as a personal memory, but one that lacked these essential attributes and invoked a 
feeling of general familiarity rather than the type of detailed attributes associated with remembered events (e.g., Gardiner, \& Richardson-Klavehn, 2000; Tulving, 1985).

Following the generation of these memories, half the participants were asked to perform an imagination task that involved picturing each memory and considering its sensory qualities. Subsequently, participants were asked to rate each memory on a seven-point rating scale ranging from 'know' to 'remember' '. The important finding was that imagery (vs. no imagery) led to 'know' memories being rated significantly closer to 'remember' on the response scale. Hyman et al., (1998) explained this as arising through the incorporation of visual-imagistic details into the original memory producing a form of false recollection.

Experiment 1 reproduced the main features of the Hyman et al. experiment but with the additional factor of the inclusion of the DVN task. Whilst performing the imagery (vs. control) task, half of each group of subjects looked at a DVN (vs. Static Visual Noise; SVN) display. If visual-imagistic information is important for altering the contents of the representation of a memory, then imagination should bring about a know-to-remember shift for 'know' memories when looking at the static noise field. However, such a shift will be reduced or eliminated when viewing a dynamic noise field.

\section{Experiment 1: Method}

\section{Design}

The design had two independent variables, each manipulated betweensubjects. The first IV was the imagery task (imagery vs. no imagery), the second IV was the dynamic noise task (DVN vs. SVN). The dependent variable was the ratings of the memories along a seven-point Likert scale ranging from $1=$ Know, to $7=$ Remember. 


\section{Participants}

The participants were 92 individuals from the Manchester Metropolitan University ${ }^{2}$. All participants took part on a voluntary basis and were recruited by the experimenters and assistants.

\section{Materials \& Apparatus}

The materials comprised a booklet that contained consent information, definitions of 'remember' and 'know' memories, task instructions, spaces to write down descriptions of two memories information and the 1-7, Likert rating scales for the DV. Separate pages were used for separate sections of information.

The DVN task was based on that described by Quinn \& McConnell (1996). It consisted of a display of $120 \times 120$ black and white squares each measuring approximately $3 \mathrm{~mm}$ by $3 \mathrm{~mm}$. In the static noise condition, the ratio of white to black squares was 50:50 and the distribution was random across the display. In the dynamic noise condition, the squares changed from black to white and vice versa in a random manner every $0.25 \mathrm{~s}$. As in the static condition, the ratio of white to black dots was maintained at 50:50. In addition, the percentage of dots changing was set to $50 \%$ (Dean et al., 2005). This produced a change rate of 7200 dots every $0.25 \mathrm{~s}$.

A computer was used to present the noise fields during phase 2 of the experiment.

\section{Procedure}

The procedure was similar to that of Hyman et al., (1998). Each participant was tested individually in a sound attenuated booth. Participants were informed that the experiment would consist of a number of phases but they were not given explicit information about the nature of each phase. 
In phase 1, the participant was given the booklet and was asked to read the section that defined autobiographical memories followed by definitions of different types of personal memory as 'remembered' and 'known'. Before moving onto the next stage, the experimenter ensured that the participant understood the definitions provided. The participant was then instructed to turn to the next page where instructions were presented for the participant to write down two memories from before the age of 10 (one memory from each of the categories: remember and know). The order was counterbalanced across the participants. Participants were asked, where possible, to include their age, the location, people involved, the activities that occurred, and other details. This procedure was similar to that of Hyman et al., (1998) and ensured that the participants were engaged in the task and understood the instructions; particularly the difference between 'remember' and 'known' memories.

In phase 2, participants were randomly allocated into one of the four experimental conditions; imagery (vs. no imagery) and DVN (vs. SVN). Those allocated to the imagery condition were asked to recall one of their two memories and form a mental image of the experience and describe any information concerning the sensory qualities of the memory that could be generated or came to mind. Whilst doing this they were asked to view a computer monitor displaying the DVN (vs. SVN) field. They were told that they should look at the noise pattern throughout the whole of the imagination stage and compliance was monitored by the experimenter. The participant did this for each of their two memories and was counterbalanced. Those participants who were in the no-imagery condition were not required to image their memory. Instead, they viewed the DVN (vs. SVN) screen and were asked a series of control questions, which were not related to the memories generated earlier. 
These were general knowledge questions selected randomly from the norms of Nelson, and Narens, (1980).

Following a short pause, participants were directed to the page of the booklet that contained the DVs and asked to rate each of their memories on the 7-point Know (1)-Remember (7) scale.

Following completion of phase 2, the participants were debriefed on the nature of the experiment and given the opportunity to ask any questions. All participants were informed of their right to withdraw from the study at any time up until the point of the analyses. They were provided with a participant number and contact information for the experimenter in case of this eventuality.

\section{Experiment 1: Results \& Discussion}

The rating scores for belief for each type of memory were placed into separate 2(imagery condition; imagery vs. no-imagery) by 2(noise condition; DVN vs. SVN) between-subjects ANOVAs. The descriptive statistics for belief can be seen in Table 1. For simple main effects results, additional analyses were undertaken to estimate the Bayes factor $(\mathrm{BF})$ for that comparison. This provides an estimation of the degree to which the results provide support for the experimental hypothesis compared to the null hypothesis.

For the present calculations, the posterior odds supporting the experimental hypothesis were used as the numerator and the posterior odds supporting the null hypothesis as the denominator $\left(\mathrm{BF}_{10}\right.$ in previous notation, e.g., Wagenmakers, et al. ,2018). With these calculations, a BF of 0.3 or less provides substantial evidence for the null hypothesis. In contrast, a factor of 3.0 or above provides evidence in favour of the experimental hypothesis. Bayes factors that fall in-between are less decisive with the weighting in favour of the experimental or null hypothesis dependent on the 
closeness of the value to either 0.3 or 3.0 (Dienes, 2011, 2014). Following the recommendations of some recent papers we used a default (half Cauchy) prior distribution with a scaling of $r=.707$ (e.g., Rouder, Speckman, Sun, Morey, \& Iverson, 2009; Wagenmakers, et al. 2018).

\section{INSERT TABLE 1 ABOUT HERE}

In relation to ratings of belief, 'remember' memories, showed no significant effect of either variable or their interaction; $F(1,88)=0.34, p=.56, \eta_{\mathrm{p}}^{2}=.004$, for noise condition, $F(1,88)=1.19, p=.29, \eta_{\mathrm{p}}^{2}=.01$, for imagination, and, $F(1,88)=$ $0.01, p=.91, \eta_{\mathrm{p}}{ }^{2}<.001$, for the interaction. For 'know' memories, the main effect of noise condition was significant, $F(1,88)=4.86, p=.03, \eta_{\mathrm{p}}^{2}=.05$. The main effect of imagination was also significant, $F(1,88)=5.53, p=.02, \eta_{\mathrm{p}}^{2}=.06$. Importantly, the interaction was significant, $F(1,88)=6.25, p=.014, \eta_{\mathrm{p}}{ }^{2}=.066$. The interaction was assessed by the use of simple main effects at each level of noise. Under SVN, imagination produced the predicted effect of increasing belief scores, $t(44)=3.92, p<$ .001 Cohen's $d=1.14, \mathrm{BF}_{10}=168.87$. However, this increase was eliminated under DVN, $t(44)=0.09, p=.92$, Cohen's $d=.03$. The $\mathrm{BF}_{10}$ for this result was 0.27 showing evidence more predominantly in favour of the null hypothesis of no difference.

Experiment 1 found that mental imagery produced a "know-to-remember" shift in the ratings of memories initially classified as known. However, this shift occurred only in the absence of visual interference produced by DVN. To the extent that DVN provides a means to interfere selectively with visual-imagistic processing (as outlined in the introduction), then the conclusion is that such forms of information contribute to the effects of imagination. This finding is important as it demonstrates that the key element of imagination is visual in nature and this forms the basis of the 
meta-cognitive evaluation of the mnemonic representation in-line with the SFM. According to that framework, monitoring processes operate on mental contents and evaluate the various qualities of the retrieved attributes including perceptual details (Johnson, et al., 1993). As perceptual and sensory details are more prevalent in actual (vs. fabricated) experiences (Johnson, et al., 1988), then 'remember' responses or judgments are more likely in representations that contain sensory and perceptual detail. If source monitoring is based upon this, then misattribution errors can result because imagination can also result in the production of perceptual details.

If the perceptual details incorporated into the representation are not from the original experience, the resulting memory can be labelled a false memory, or to be more precise, a false recollection as the recollective details were not present in the original retrieval attempt or accurately represent the original experience. However, this is not the only explanation. To the extent that repeated retrieval results in hypermnesic effects (Erdelyi \& Becker, 1974; Roediger \& Thorpe, 1978), then the perceptual details incorporated into post-imagery representations may reflect sensory features that were in fact originally encoded. Thus, when originally recalling a 'known' memory (in phase 1), many perceptual attributes may be missing. However, following repeated recall with instructions to imagine (phase 2), may result in the activation of the original sensory records that are then incorporated into the final representation that is used as basis for judgement. The findings from Experiment 1 cannot decide between either of these explanations. However, the important point is that whatever the processes involved (imagination of visual features not originally encoded vs. reactivation of encoded features during imagination), they are sensitive to a technique that interferes with the processing of visual-imagistic information. 


\section{Experiment 2. Imagination \& DVN effects on judgements of unlikely childhood} events.

Experiment 2 extends the first experiment by examining the effects of imagination and DVN using a different paradigm that incorporated events that were extremely unlikely to have occurred. In addition, it assessed the effects of imagination and DVN on both the believability and vividness responses. The design was based on that of Mazzoni and Memon (2003) in which participants were asked to indicate their degree of belief in a number of event statements that could/could-not have occurred in childhood before the age of ten.

One of these was labelled a non-occurring event to denote that the incident described could not occur. This event was "having a nurse remove a skin sample from the little finger". In the United Kingdom, where the Mazzoni and Memon (and the current) experiments were conducted, no such test takes place. Another event was one that was very likely to have occurred for the majority of participants and was "having a milk tooth extracted by a dentist". This was labelled as a relatively frequent event as it is possible that many participants could believe or find this event highly probable. Other events were taken from the Life Events Inventory (LEI) that comprises a number of statements referring to plausible childhood life events such as "made a 911 call" and "won a stuffed animal at the carnival" (Garry et al., 1996). In the usual administration of the LEI, participants are asked to rate their belief that these events occurred to them before the age of ten. Mazzoni and Memon (2003) found that imagination increased belief for the events including the non-occurring incident.

The question posed for Experiment 2 was whether that effect was dependent on the processing of visual-imagistic information. To assess this, an adapted version of the Mazzoni and Memon (2003) experiment was used that involved a number of 
changes. Firstly, the delay between the imagination and final test phase was reduced. Secondly, the pre-testing phase for the event items was not used because of concerns about participants merely attempting to recall earlier pre-test scores in the final rating phase (especially under a reduced delay). Thirdly, the imagination manipulation in Experiment 2 was between-subjects rather than within-subjects. All three of these alterations increased consistency with experiment 1 . Finally, in the Mazzoni \& Memon experiment, the no-imagination control task involved reading a short text describing the event. In Experiment 2, this was changed to listening; reading could not be used as participants would be viewing the DVN (vs. SVN) screen.

Accordingly, participants were exposed to a range of event scenarios, including the non-occurring event and others taken from the LEI. Participants were asked to imagine or listen to these events under conditions of either SVN or DVN and then indicate their judgments about these 'memories' in terms of belief and vividness. Vividness, although not included in the Mazzoni and Memon (2003) experiment, was used here as this has often been associated with the recollective or 'reliving' qualities of personal memory (Cabeza \& St Jacques, 2007; Rubin, 2006) and can provide some indication of whether the memory contains sensory qualities usually found in experienced autobiographical events. It was hypothesised that imagination would increase belief and vividness judgements but only under conditions of SVN.

\section{Experiment 2: Method}

\section{Design}

The design had two independent variables both manipulated between-subjects. The first was the noise condition with two levels; DVN vs. SVN. The second was the encoding of the events, with two levels; listening and imagination. The dependant 
variables were belief and vividness of the memory. Each of the variables were measured using an 8-point Likert scale indicating participants level of belief/vividness of the memory ranging from 1 to 8 . For belief, this spanned from $1=$ definitely did not happen to me to $8=$ definitely did happen to me. For vividness, this spanned $1=$ not at all vivid to $8=$ very vivid.

\section{Participants}

The participants were 120 individuals from the Manchester Metropolitan University who took part on a voluntary basis and were recruited by experimental assistants via opportunity sampling.

\section{Materials \& Apparatus}

The materials comprised of a booklet that contained separate sections relating to different phases of the experiment. For phase 1, the booklet provided general details about the experiment and spaces for demographic information. The general details indicated that the experiment involved a number of phases related to personal memory and imagination. No information was provided about the particulars of the experiment.

For phase 2, the booklet had a separate section that contained the response variables. These were the event names (but not the descriptions) pertaining to the three event narratives (outlined below). The narratives were not presented in the booklet because these were to be described to subjects in an auditory manner. These titles were followed by two 8-point Likert scales for belief and for vividness. For belief, the scale ranged from 1 denoting 'definitely did not happen to me' to 8 indicating 'definitely did happen to me'. For vividness the scale ranged from 1 indicating 'not at all vivid' to 8 designating 'very vivid'. Thus, each event statement was followed by the two response scales. 
A set of 3 event narratives (descriptions) were developed, pertaining to the non-occurring event of Mazzoni and Memon's (2003) study (having a nurse remove a skin sample from the little finger) and 2 events taken from the LEI of Garry et al (1996). The latter included "Got in trouble for calling 999" and "Won a stuffed animal at the fun fair". The two LEI events were counterbalanced so that one appeared during the imagination/listen phase and the other appeared only on the final test. This was done to assess if recent imagination or viewing DVN had an effect on responding even without prior exposure to the event. The event narratives consisted of a title (the name of the event) and a short story-like account of the event itself including event related details.

The narratives were created as a result of pilot testing in which a pool of 10 participants were asked to describe the typical features of these events and the experiences that may have occurred for each event statement. That the non-occurring event was not experienced by the participants was not considered to be problematic as the descriptions that were elicited required only the generation of information that might have been plausibly encountered. Thus, the production of such a scenario, could be achieved by making use of pre-exiting knowledge about nurses, examinations and other medical themes. On the basis of the information generated, common features were then identified by the experimenters from which short narratives were created. The event narratives were constructed to be of similar length and recorded into audio files that lasted approximately $60 \mathrm{~s}$ each (including pauses). These narratives were not printed in the booklets but only presented in audio format in phase 2 of the experiment. The DVN task was the same as that described in Experiment 1. 


\section{Procedure}

The procedure broadly followed that of Mazzoni and Memon (2003). All participants were tested individually in a sound attenuated booth and were allocated randomly to the conditions. Participants were informed that the experiment would consist of a number of phases but they were not given explicit information of the nature of those.

In phase 1, the participant was provided with the booklet relevant to their assigned condition. It contained general information about the experiment (such that it involved a number of phases related to memory and the ability to imagine) but did not reveal any information pertaining to the particulars or more precise details.

Following this, participants were instructed to turn to a separate page in the booklet and read the instructions that indicated that they would be required to view a computer monitor displaying either the SVN or DVN field depending on the condition to which they had been allocated. Additionally, they were informed that whilst viewing the screen, they would be asked to either imagine or simply listen to the event narrative.

The experimenter then asked the subjects to face the computer screen displaying either SVN or DVN. Once the participants were viewing the display, the audio files of the event narratives were started. For those in the listen condition, the instructions requested listening carefully to the narrative while maintaining visual gaze on the screen. For those allocated to the imagine condition, the instructions requested imagining the scenario and picturing as much of the content as possible in their minds eye. Each event narrative played for approximately $60 \mathrm{~s}$. Compliance with instructions to maintain gaze on the screen was observed by the experimenter. The order of presentation of the narratives was counterbalanced. 
Following exposure to the two event narratives, there was a short pause (as in experiment 1), and then participants were asked to turn to the section of the booklet that contained the DVs. For each narrative, a separate page was used. Each narrative was indicated by its name (not the description of the event narrative) followed by two 8-point scales to rate level of belief and vividness for each narrative.

Once the participant had completed the set of scales, they were provided with participant numbers, a contact email address and were debriefed and informed of their right to withdraw at any point up to a particular date.

\section{Experiment 2: Results \& Discussion}

The analyses for each memory type and for each DV were entered into separate 2(imagery condition; imagery vs. no-imagery) by 2(noise condition; DVN vs. SVN) between-subjects ANOVAs. The descriptive statistics can be seen in Table 2.

\section{INSERT TABLE 2 ABOUT HERE}

For belief in the non-occurring event, the main effect of noise condition was significant, $F(1,116)=8.37, p=.005, \eta_{\mathrm{p}}^{2}=.07$. The main effect of imagination was not significant, $F(1,116)=1.06, p=.30, \eta_{\mathrm{p}}^{2}=.019$, however the interaction was significant, $F(1,116)=4.87, p=.029, \eta_{\mathrm{p}}^{2}=.04$.

The interaction was assessed by the use of simple main effects at each level of the noise condition. In the SVN condition, imagination increased ratings of belief, $t(58)=2.08, p=.04$, Cohen's $d=0.53, \mathrm{BF}_{10}=3.04$. Under DVN, no effect of imagination was observed, $t(58)=0.94, p=.35$, Cohen's $d=0.26, \mathrm{BF}_{10}=0.17$. The direction of the means was also in the opposite direction.

For vividness ratings for the non-occurring event the main effect of imagination was close to significance, $F(1,116)=3.36, p=.07, \eta_{\mathrm{p}}{ }^{2}=.03$, the main effect of noise was significant, $F(1,116)=8.74, p=.004, \eta_{\mathrm{p}}^{2}=.07$, and the 
interaction was close to conventional levels of significance, $F(1,116)=3.36, p=.07$, $\eta_{\mathrm{p}}^{2}=.03$

Given the importance of the interaction, this was assessed further by the use of simple main effects at each level of the noise condition. In the SVN condition, imagination increased ratings of vividness $t(58)=2.27, p=.03$, Cohen's $d=0.58$, $\mathrm{BF}_{10}=4.27$. Under DVN, no effect of imagination was observed, $t(58)=0.001, p>$ .05 , Cohen's $d=0.01, \mathrm{BF}_{10}=0.31$.

For belief in the LEI when exposed, this produced a main effect of noise condition, $F(1,116)=29.22, p<.001, \eta_{\mathrm{p}}^{2}=.20$. The main effect of imagination was not significant, $F(1,116)=0.68, p=.41, \eta_{\mathrm{p}}{ }^{2}=.006$. However, the interaction was significant, $F(1,116)=12.12, p=.001, \eta_{\mathrm{p}}{ }^{2}=.095$.

Simple main effects were conducted at each level of the noise condition. This produced a significant effect of imagination under SVN, $t(58)=3.10, p=.003$, Cohen's $d=0.80, \mathrm{BF}_{10}=24.97$, indicating higher ratings of belief following imagination. Under DVN, the difference was smaller, in the opposite direction and only close to conventional levels of significance, $t(58)=1.85, p=.07$, Cohen's $d=$ $0.37, \mathrm{BF}^{10}=0.01$

For ratings of vividness in the LEI when exposed, this produced a main effect of noise condition, $F(1,116)=35.91, p<.001, \eta_{\mathrm{p}}^{2}=.24$. The main effect of imagination was not significant, $F(1,116)=2.61, p=.11, \eta_{\mathrm{p}}{ }^{2}=.022$. However, the interaction was significant, $F(1,116)=13.06, p<.001, \eta_{\mathrm{p}}^{2}=.10$.

Simple main effects were conducted at each level of the noise condition. This produced a significant effect of imagination under SVN, $t(58)=3.44, p=.001$, Cohen's $d=0.87, \mathrm{BF}_{10}=57.09$, indicating increased ratings of vividness following 
imagination. However, the difference was not significant under DVN, $t(58)=1.54, p$ $=.13$, Cohen's $d=0.4, \mathrm{BF}_{10}=0.11$, and the difference was in the opposite direction.

For belief in the LEI when not exposed (baseline condition), this produced no main effect of noise condition, $F(1,116)=0.30, p=.58, \eta_{\mathrm{p}}^{2}=.003$, no main effect of imagination, $F(1,116)=1.12, p=.29, \eta_{\mathrm{p}}^{2}=.009$, and no interaction, $F(1,116)=$ $0.09, p=.77, \eta_{\mathrm{p}}{ }^{2}=.001$. For vividness ratings in the LEI when not exposed this produced no main effect of noise condition, $F(1,116)=0.45, p=.50, \eta_{\mathrm{p}}{ }^{2}=.004$, no main effect of imagination, $F(1,116)=0.002, p=.96, \eta_{\mathrm{p}}{ }^{2}<.001$, and no interaction, $F(1,116)=0.05, p=.82, \eta_{\mathrm{p}}^{2}<.001$.

Additional analyses were performed to assess the relationship between the vividness and belief scores in each of the conditions for each event type ${ }^{3}$. The Pearson product moment correlations can be seen in Table 3.

\section{INSERT TABLE 3 ABOUT HERE}

All relationships were positive and significant to below $p<.001$ (largest $p$ was for the LEI baseline under SVN and listen condition). To assess the significance of any differences between the correlation values across the conditions, Fisher's r-to-z transformations were computed. To avoid an excessive number of comparisons, only two contrasts (imagination vs. listen) were assessed for each event-type for each level of the noise conditions and was thus in-line with the simple comparisons made following the ANOVAs. The results can be seen on the right half of Table 3. The only difference that achieved conventional significance was that for the LEI exposed event under imagination, in which the relationship between belief and vividness was marginally higher.

Overall, Experiment 2 found results congruent with Experiment 1. Namely, imagining an event increased belief in that event and reported vividness. However, the 
effect was reduced in the presence of a DVN field. Although the magnitude of the inflation effect was smaller for the non-occurring event, the difference between listen and imagination was eliminated also by dynamic noise. Once more, the important point is that the inflation effect was dependent on visual-imagistic processing; when such processing attempts were undermined (by DVN) then the inflation effect disappeared.

The use of a non-exposed memory statement (LEI baseline) was used to assess the impact that any recent imagination or exposure to a DVN field might have upon responding even when the event itself had not been exposed in the initial phase. If these factors were to influence ratings for the baseline event, this could indicate the operation of response bias. However, neither effects of imagination or DVN were found, indicating that the influence of both imagination and dynamic noise are specific to the contents of the representations activated during phase 1 of the procedure.

The relationship between belief and vividness was significant across all conditions and is similar to other reports that demonstrate positive relationships between belief and recollection (e.g., Rubin, 2005; Rubin, Schrauf, \& Greenberg, 2003). Possibly this reflects the influence of vividness on the beliefs about accuracy and is thus consistent with the SFM; the recall of particular (vivid) details is considered diagnostic of having experienced an event and this serves to make it more believable (Kelley, \& Sahakyan, 2003). However, caution needs to be exercised with the interpretation of directed of influence as these findings were only correlational.

\section{General Discussion}

\section{General overview \& summary}


The present research found that imagination can influence judgements about the personal past in terms of remembering (vs. knowing) (Experiment 1) and ratings of belief/vividness of improbable and plausible events (Experiment 2). Previous work has suggested that the effects of imagination on judgements such as these reflect the incorporation of visual-imagistic representations into the event or memory narrative. The current findings support this hypothesis because techniques that are known to impair visual-imagistic processing (i.e., DVN) reduce the effects of imagination on such meta-cognitive judgements. This was found across two experiments that employed different materials and measurements of belief and judgement of personal memories. Overall, the current results indicate that the incorporation of visualimagistic information into a "possible" memory narrative, can enhance the believability and vividness of that narrative. This outcome is explicable in terms of previous conceptualisations of false autobiographical memory and the mechanisms that are influenced by dynamic noise fields.

\section{Theoretical accounts of effects}

The findings obtained in both experiments can be understood from the perspective of the SMF and the mechanisms that are hypothesised to underlie DVN effects on memory. In relation to the former, the SMF explains memory as the result of a number of processes including the retrieval of mental contents, their appraisal, monitoring and judgements about the origins of the experience. If visual-imagistic information is retrieved, then it is more likely that the experience will be considered a true memory. This comes about because the qualities of the retrieved contents are appraised and appropriate judgements derived in-line with the appraisal. In this case, because on average, real experiences contain more perceptual detail, then an external source attribution will occur. Although this may be accurate in many circumstances, it 
will sometimes be in error if the perceptual details were derived from other sources, such as vivid imagining. In the case of the experiments reported here, those details could originate from a combination of 'pure' imagination (that bore no grounding in the original experience) and perceptual details of the original or similar events that were reactivated during imagination. For similar events, these visual attributes can then be imported to the target memory making that memory seem more vivid and real (Lyle \& Johnson, 2006). With regard to the first, this might account for the effects found in relation to improbable events. This could also apply to known memories in combination with the reactivation of encoded visual details. In both cases however, DVN reduces the effect because it interferes with the generation of imagistic representations.

The SMF provides a general perspective for considering true and false memory, however, more particular frameworks have been proposed within the context of false autobiographical memory research. For example, Hyman et al., (1998), contend that false personal memories are constructed following a sequence of processes involving: (i) accepting a possible memory or narrative as plausible, (ii) elaborating on that narrative by the incorporation of additional (including sensory) details, and finally (iii) misattributing the imagined construction to an actual event (that is, a source misattribution error accountable within the SMF).

From the perspective of the current work, the target events in Experiment 1 were self-selected and thus possessed at least some degree of personal plausibility. For Experiment 2, the events were selected by the experimenter to at least possess some degree of general plausibility (even the non-occurring event had some degree of plausibility). Consequently, the effects of imagination (and the influence of DVN) as reported here relate to event constructions that have some degree of credibility or 
believability in the first instance. In this context, it is unlikely that DVN influences judgements of plausibility per-se. Rather, it is within the second stage of Hyman et al's framework that DVN is likely to exert its effects; through interfering with the elaboration or creation of a narrative. More specifically, by interfering with the generation of visual-imagistic information, subsequent source monitoring decisions are made easier as less event-specific sensory details are present in the final representation.

The use of DVN in the context of visual imagination inflation effects provides some theoretical leverage on the nature of such imagery effects. Imagery and perceptual information can be represented in terms of abstract codes, that themselves do not possess the properties of images or in a form that is non-propositional or depictive (picture-like) (Pearson, Naselaris, Holmes, \& Kosslyn, 2015). DVN has been shown to exert effects primarily in tasks that require the generation of mental images during encoding or retrieval in their non-propositional form whilst being processed by the visual buffer (Pearson, 2001; Quinn \& McConnell, 2006). According to this account, the visual buffer is responsible for the representation of conscious visual images that are short-lived unless refreshed and can be acted upon by the central executive according to current goals (Quinn \& McConnell, 2006).

Consequently, the conclusion to be drawn from the current results is that visual-imagery based inflation effects are initially dependent on representations processed by the visual buffer. During this period, these representations are vulnerable to external visual interference and hence disruption by a DVN field. However, as these representations decay rapidly, the longer-term effects must be due to their storage in alternative forms. 
The introduction outlined a distinction between episodic and semantic autobiographical memories. The former includes event-specific details that are less prevalent in the latter (Renoult, Davidson, Palombo, Moscovitch, \& Levine, 2012). This distinction is also embodied in other theoretical conceptions. For example, the difference between 'remember' and 'know' responses (Conway, Collins, Gathercole, \& Anderson, 1996; Tulving, 1985), or between 'memory' and 'belief' (Mazzoni, \& Kirsch, 2002). The first of each of these distinctions refers to remembered experiences with details of the occurrence of the events. The second refers to knowledge without recollection or to the degree of certainty regarding the occurrence of an event (e.g., rating the likelihood that some experience or event has really happened). Thus, knowing and beliefs, in this context are like personal semantic memories that are retrieved without detail. It has been argued that many of the experiments into false autobiographical memories are mainly concerned with the latter (Brewin \& Andrews, 2016; Mazzoni, \& Kirsch, 2002).

Considered within this context, the false autobiographical memories produced in previous studies on imagination inflation, and the ones created here, may more accurately be described as false beliefs or false know responses about the self. For example, in Experiment 1, although there was shift from know to remember, the inflated ratings only just reached the midpoint of the scale and were lower than the ratings for genuine remember type memories. In Experiment 2, although there was some shift towards a higher scale rating (e.g., this event did happen to me), the responses following imagination were still low; typically below the midpoint of the scale with the exception of the imagination and SVN condition for the LEI exposed memory. Accordingly, it remains to be seen whether imagination techniques can produce effects that are more episodic-like and if these can also be reduced by DVN. 


\section{Future research considerations}

In both of the experiments reported here, DVN was applied during the imagination phase. Previous, work has demonstrated that DVN can indeed act upon the retrieval of imagistic representations (Dean et al., 2005; Parker \& Dagnall, 2009) and future work might want to consider the application of DVN at the retrieval or judgement phase when participants actually make their responses about the believability or vividness of their memories.

In the current experiments the delay between the imagination and judgement phase was relatively short. As previous work has used both short (e.g., Hyman et al., 1998; Libby, 2003) and longer delays (e.g., Garry et al., 1996; Mazzoni \& Memon, 2003), an important consideration would be to assess the extent to which the effects of DVN continue over longer delays.

In this research, the focus was on the role of visual imagination as this is of special importance due to the predominance of visual processing in models of autobiographical memory (e.g., Greenberg, \& Rubin, 2003; Greenberg \& Knowlton, 2014; Rubin, 2006). However, as autobiographical memories are complex multimodal representations (Cabeza \& St Jacques, 2007), it will be valuable for future work to consider whether modality-specific interference can be found.

In both of the current experiments, the descriptions of the imagined information during the imagery trials was not recorded but could be considered in future work ${ }^{4}$. Particularly, with the intent to assess whether the amount and type of descriptions differ under DVN (vs. SVN) conditions, and how this relates to the magnitude of inflation effects.

\section{Conclusion}


The two experiments described here indicate that DVN can reduce the magnitude of imagery inflation effects. This was found using different experimental procedures and different memory elicitation/induction techniques. The findings extend the range of situations in which DVN effects can be found and demonstrate that imagination inflation effects are, in part, dependent upon the generation and use of visual imagery-based processes and representations. 


\section{References}

Allen, B. P., \& Lindsay, D. S. (1998). Amalgamations of memories: Intrusion of information from one event into reports of another. Applied Cognitive Psychology, 12, 277-285.

Anderson, R. J., Dewhurst, S. A., \& Dean, G. M. (2017). Direct and generative retrieval of autobiographical memories: The roles of visual imagery and executive processes. Consciousness \& Cognition, 49, 163-171.

Bays, R. B., Zabrucky, K. M., \& Foley, M. A. (2015). Imagery induction processes differentially impact imagination inflation. Imagination, Cognition, and Personality, 35, 5-25.

Brewin, C. R., \& Andrews, B. (2016). Creating memories for false autobiographical events in childhood: A systematic review. Applied Cognitive Psychology, 31, 2-23.

Cabeza, R., \& St Jacques, P. (2007). Functional neuroimaging of autobiographical memory. Trends In Cognitive Sciences., 11, 219-27.

Conway, M. A. (2005). Memory \& the self. Journal of Memory \& Language, 53, $594-628$.

Conway, M. A., Collins, A. F., Gathercole, S. E., \& Anderson, S. J. (1996).

Recollections of true and false autobiographical memories. Journal of Experimental Psychology: General, 125, 69-95.

Conway, M.A., \& Pleydell-Pearce, C.W. (2000). The construction of autobiographical memories in the self-memory system. Psychological Review, 107, 261-288. 
Crombag, H. F. M., Wagenaar, W. A., \& van Koppen, P. J. (1996). Crashing memories and the problem of "source monitoring". Applied Cognitive Psychology, 10, 95-104.

Dean, G. M., Dewhurst, S. A., Morris, P. E., \& Whittaker, A. (2005). Selective interference with the use of visual images in the symbolic distance paradigm. Journal of Experimental Psychology: Learning, Memory \& Cognition, 31, $1043-1068$.

Dent, K. (2009). Dynamic visual noise affects visual short-term memory for surface color, but not spatial location. Experimental Psychology, 57, 17 - 26.

Dienes, Z. (2011). Bayesian versus orthodox statistics: which side are you on? Perspectives in Psychological Science, 6, 274-290.

Dienes, Z. (2014). Using Bayes to get the most out of non-significant results. Frontiers in Psychology, 5:781. doi: 10.3389/fpsyg.2014.00781

Dunn, J. C. (2004). Remember-know: A matter of confidence. Psychological Review, $11,524-542$.

Erdelyi, M.H., \& Becker, J. (1974). Hypermnesia for pictures: Incremental memory for pictures but not words in multiple recall trials. Cognitive Psychology, 6, $159-171$.

Frenda, S. J., Knowles, E. D., Saletan, W. \& Loftus, E. F. (2013) False memories of fabricated political events. Journal of Experimental Social Psychology, 49, $280-86$.

Gardiner, J. M., \& Richardson-Klavehn, A. (2000). Remembering and knowing. In E. Tulving \& F. I. M Craik (Eds.), The Oxford handbook of memory (pp. 229244). Oxford: Oxford University Press. 
Garry, M., Manning, C. G., Loftus, E. F., \& Sherman, S. J. (1996). Imagination inflation: Imagining a childhood event inflates confidence that it occurred. Psychonomic Bulletin and Review, 3, 208-214.

Goff, L. M., \& Roediger, H. L. (1998). Imagination inflation for action events: Repeated imaginings lead to illusory recollections. Memory \& Cognition, 26, $20-33$.

Gonsalves, B., Reber, P. J.,Gitelman,D.R., Parrish, T. B.,Mesulam,M.M.,\&Paller, K. A. (2004). Neural evidence that vivid imagining can lead to false remembering. Psychological Science, 15, 655-660.

Greenberg, D.L., \& Knowlton, B.J. (2014). The role of visual imagery in autobiographical memory. Memory \& Cognition, 42, 922-934.

Greenberg, D.L. and Rubin, D.C. (2003) The neuropsychology of autobiographical memory. Cortex, 39, 687-728

Horselenberg, R., Merckelbach, H., Muris, P., Rassin, E., Sijsenaar, M., \& Spaan, V. (2000). Imagining fictitious childhood events: The role of individual differences in imagination inflation. Clinical Psychology \& Psychotherapy, 7, $128-137$.

Hyman, I. E., Jr., Gilstrap, L. L., Decker, K., \& Wilkinson, C. (1998). Manipulating remember and know judgements of autobiographical memories: An investigation of false memory creation. Applied Cognitive Psychology, 12, $371-386$.

Johnson, M. K., Foley, M. A., Suengas, A. G., \& Raye, C. L. (1988). Phenomenal characteristics of memories for perceived and imagined autobiographical events. Journal of Experimental Psychology: General, 117, 371-376. 
Johnson, M. K., Hashtroudi, S., \& Lindsay, D. S. (1993). Source monitoring. Psychological Bulletin, 114, 3-28.

Johnson, M. K., Raye, C. L., Wang, A. Y., \& Taylor, T. H. (1979). Fact and fantasy: The roles of accuracy and variability in confusing imaginations with perceptual experiences. Journal of Experimental Psychology. Human Learning and Memory, 5, 229-240.

Kemps, E., \& Andrade, J. (2012). Dynamic visual noise reduces confidence in shortterm memory for visual information. Cognitive Processes, 13, 183-188.

Kelley, C. M., \& Sahakyan, L. (2003). Memory, monitoring and control in attainment of memory accuracy. Journal of Memory and Language, 48, $704-$ 721.

Kosslyn, S. M., Thompson, W. L., \& Ganis, G. (2006). The case for mental imagery. New York: Oxford University Press.

Levine, B. (2004). Autobiographical memory and the self in time: Brain lesion effects, functional neuroanatomy, and lifespan development. Brain \& Cognition, 55, 54-68.

Libby, L. K. (2003). Imagery perspective and source monitoring in imagination inflation. Memory \& Cognition, 31, 1072-1081

Lindner, I. \& Echterhoff, G. (2015). Imagination inflation in the mirror. Can imagining others'actions induce false memories of self-performance? Acta Psychologica, 158, 51-60.

Lindsay, D. S., Johnson, M. K., \& Kwon, P. (1991). Developmental changes in memory source monitoring. Journal of Experimental Child Psychology, 52, 297-318. 
Loftus, E. F., \& Davis, D. (2006). Recovered memories. Annual Review of Clinical Psychology, 2, 469-498.

Lyle, K. B., \& Johnson, M. K. (2006). Importing perceived features into false memories. Memory, 14, 197-213.

Lyle, K. B., \& Johnson, M. K. (2007). Source misattributions may increase the accuracy of source judgments. Memory \& Cognition, 35, 1024-1033.

Marsh, B. U., Pezdek, K., \& Lam, S. T. (2014). Imagination perspective affects ratings of the likelihood of occurrence of autobiographical memories. Acta Psychologica, 150, 114-119.

Mather, M., Henkel, L. A., \& Johnson, M. K. (1997). Evaluating characteristics of false memories: Remember/Know judgments and memory characteristics questionnaire compared. Memory \& Cognition, 25, 826-837.

Mazzoni, G., \& Kirsch, I. (2002). Autobiographical memories and beliefs: A preliminary metacognitive model. In T. J. Perfect \& B. L. Schwartz (Eds.), Applied metacognition (pp. 121-145). Cambridge, UK: Cambridge University Press.

Mazzoni, G., \& Memon, A. (2003). Imagination can create false autobiographical memories. Psychological Science, 14, 186-188.

Nelson, T. O., \& Narens, L. (1980). Norms of 300 general-information questions: Accuracy of recall, latency of recall, and feeling-of-knowing ratings. Journal of Verbal Learning \& Verbal Behavior, 19, 338-368.

Odegard, T. N., \& Lampinen, J. M. (2004). Memory conjunction errors for autobiographical events: More than just familiarity. Memory, 12, 288-300. 
Okado, Y., \& Stark, C. (2003). Neural processing associated with true and false memory retrieval. Cognitive, Affective, \& Behavioral Neuroscience, 3, 323334.

Parker, A., \& Dagnall, N. (2009). Concreteness effects revisited The influence of dynamic visual noise on memory for concrete and abstract words. Memory, 17, $397-410$.

Pearson, D.G., (2001). Imagery and the visuo-spatial sketchpad. In J Andrade, (Ed.), Working Memory in Perspective (pp. 33-59). Psychology Press: Hove.

Pearson J., Naselaris T., Holmes E., Kosslyn S. (2015). Mental imagery: functional mechanisms and clinical applications. Trends in Cognitive Sciences, .19, 590602.

Quinn, J. G., \& McConnell, J. (1996). Irrelevant pictures in visual working memory. Quarterly Journal of Experimental Psychology, 49A, 200 - 215.

Quinn, J. G., \& McConnell, J. (2006). The interval for interference in conscious visual imagery. Memory, 14, $241-252$.

Renoult, L., Davidson, P. S., Palombo, D. J., Moscovitch, M., \& Levine, B. (2012). Personal semantics: at the crossroads of semantic and episodic memory. Trends in Cognitive Sciences, 16, 550-558.

Roediger, H. L., \& McDermott, K. B. (1995). Creating false memories: Remembering words not presented on lists. Journal of Experimental Psychology: Learning, Memory \& Cognition, 21, 803 - 814.

Roediger, H.L., III, \& Thorpe, L.A. (1978). The role of recall time in producing hypermnesia. Memory \& Cognition, 6, 296-305. 
Rouder, J. N., Speckman, P. L., Sun, D., Morey, R. D., \& Iverson, G. (2009). Bayesian $t$ tests for accepting and rejecting the null hypothesis. Psychonomic Bulletin \& Review, 16, 225-237.

Rubin, D. C. (2005). A basic-systems approach to autobiographical memory. Current Directions in Psychological Science, $14,79-83$.

Rubin, D.C. (2006). The basic-systems model of episodic memory. Perspectives in Psychological Sciences 1, 277-311.

Rubin, D.C., Schrauf, R.W., \& Greenberg, D.L. (2003). Belief and recollection of autobiographical memories. Memory \& Cognition, 31, 887-901.

Schacter, D. L. (1999). The seven sins of memory: insights from psychology and cognitive neuroscience. American Psychologist, 54, 182 - 203.

Sugimori, E., Mitchell, K. J., Raye, C. L., Greene, E. J., \& Johnson, M. K. (2014). Brain mechanisms underlying reality monitoring for heard and imagined words. Psychological Science, 25, 403- 413.

Thomas, A. K., Bulevich, J. B., \& Loftus, E. F. (2003). Exploring the role of repetition and sensory elaboration in the imagination inflation effect. Memory \& Cognition, 31, 630-640.

Thomas, A. K., \& Loftus, E. F. (2002). Creating bizarre false memories through imagination, Memory \& Cognition, 30, 423-431.

Tulving, E. (1985). Memory and consciousness. Canadian Psychology, 25, 1-12.

Wagenmakers, E. J., et al. (2018). Bayesian inference for psychology, part II: Example applications with JASP. Psychonomic Bulletin and Review, 25, 5876. 
Yonelinas, A. P., Aly, M., Wang, W. C., \& Koen, J. D. (2010). Recollection and familiarity: Examining controversial assumptions and new directions. Hippocampus, 20, 1178-1194. 


\section{Footnotes}

1. In Hyman et al. (1998) and Experiment 1 of the current paper, the distinction between remembering and knowing was treated initially in a dichotomous manner (generating memories that were either 'remembered' or 'known') and later in a continuous manner (using the 7-point rating scale with 1 as known and 7 as remember). For readers familiar with dual-process models of memory this 'cross-classification' and usage of such terms may seem unwarranted. This is because extant theorising about the basis of memory has treated these two forms of memory as qualitatively distinct (dual process) (Yonelinas, 2002), or varying along a continuum of memory trace strength (single process) (Dunn, 2004).

However, the purpose of the current work (and Hyman et al., 1998) was not to demarcate between dual (vs. single) process accounts of memory. Rather, the initial memory generation and classificatory task, was to allow the production of different types of memory that varied in terms of the details that each contained. For a memory that is generated as 'known' the rating of this will likely vary along a continuum depending upon the relative familiarity of the memory. Irrespective of this, the ratings will be more towards the 'know' end of the continuum. This was the outcome in Hyman et al., (1998) and Experiment 1 of the present research.

For memories classified as 'remembered' it might be conjectured that all ratings should be designated a maximum of 7 on the rating scale (because if a memory is indeed 'remembered' and thus rich in episodic detail, then it should not receive a rating score of less than 7). However, remembering (recollection) is not necessarily an all or none process (Yonelinas, Aly, Wang, \& Koen, 2010). Thus, individuals can remember more or less of the precise episodic details of an event and this will translate into variations in the responses on the rating scale; albeit with all such memories being rated as high on the response sale continuum (Yonelinas et al., 2010). This was likely reflected in Hyman et al., (1998) and Experiment 1 of the current work in which the mean of the 
'remembered' memories was less than 7 and rated as 5.79 (see the upper half of Table 1 in the result section).

2. Sample sizes were determined based on previous DVN research. The stopping rule was when we reached a minimum of 20 per-between subject group or the end of an academic year, whichever produced the most. The maximum was 30.

3. These analyses were not planned but were suggested by a reviewer. We performed these as it was felt that such relationships might prove to be of interest in the context of this and prior work on autobiographical memory.

4. For Experiment 2, this was partly precluded by the fact that the participant was listening to the recorded description of the events. Of course, it could have been possible to play the event narrative via headphones and record any verbal elaborations. However, doing this may have made the task unduly complex. 
TABLE 1

Mean belief rating (and SD) as a function of interference condition, memory type and imagination condition.

\begin{tabular}{lll}
\hline & \multicolumn{2}{c}{ Interference Condition } \\
\cline { 2 - 3 } $\begin{array}{l}\text { Memory Type } \\
\text { \& Imagination Condition }\end{array}$ & Static & Dynamic \\
\hline \multirow{2}{*}{ Remember } & & \\
& & \\
Imagination & $5.74(0.86)$ & $5.65(0.93)$ \\
No Imagination & $5.95(0.87)$ & $5.83(0.87)$ \\
Know & & \\
Imagination & $4.17(1.07)$ & $2.78(1.28)$ \\
No Imagination & $2.74(1.38)$ & \\
\hline
\end{tabular}


TABLE 2

Mean belief \& vividness rating (SD) as a function of interference condition, memory type and imagination condition.

\begin{tabular}{lcc}
\hline & \multicolumn{2}{c}{ Interference Condition } \\
\cline { 2 - 3 } $\begin{array}{l}\text { Memory Type } \\
\text { \& Imagination Condition }\end{array}$ & Static & Dynamic \\
\hline Non-occurring & Belief & \\
Imagination & & $1.67(1.56)$ \\
No Imagination & $3.40(2.34)$ & $2.10(1.74)$
\end{tabular}

LEI exposed

$\begin{array}{lll}\text { Imagination } & 5.83(1.86) & 2.60(1.94) \\ \text { No Imagination } & 4.26(2.04) & 3.57(2.11)\end{array}$

LEI baseline

$\begin{array}{lll}\text { Imagination } & 3.57(1.83) & 3.23(2.21) \\ \text { No Imagination } & 3.87(2.12) & 3.77(2.42)\end{array}$

Vividness

Non-occurring

$\begin{array}{lll}\text { Imagination } & 3.13(2.43) & 1.56(1.47) \\ \text { No Imagination } & 1.93(1.57) & 1.57(1.50)\end{array}$

LEI exposed

$\begin{array}{lll}\text { Imagination } & 5.70(1.90) & 2.33(1.71) \\ \text { No Imagination } & 3.87(2.22) & 3.03(1.81)\end{array}$

LEI baseline

$\begin{array}{lll}\text { Imagination } & 3.30(2.05) & 2.97(2.12) \\ \text { No Imagination } & 3.20(2.02) & 3.03(1.97)\end{array}$


TABLE 3

Pearson product moment correlations between belief and vividness ratings as a function of experimental condition and Fisher's r-to-z transformation statistics for correlation comparisons.

\begin{tabular}{llclcc}
\hline & & Interference Condition & & \multicolumn{2}{c}{ Test Statistic } \\
\cline { 2 - 3 } $\begin{array}{l}\text { Event Type } \\
\text { \& Imagination Condition }\end{array}$ & Static & Dynamic & & $z$ & $p$ \\
\hline
\end{tabular}

Non-occurring

$\begin{array}{lllll}\text { Imagination } & .96 & .91 & 1.54 & .12 \\ \text { No Imagination } & .92 & .91 & 0.23 & .82\end{array}$

LEI exposed

$\begin{array}{lllll}\text { Imagination } & .86 & .95 & 1.98 & .05 \\ \text { No Imagination } & .93 & .83 & 1.73 & .08\end{array}$

LEI baseline

$\begin{array}{lllll}\text { Imagination } & .84 & .93 & 1.61 & .12 \\ \text { No Imagination } & .70 & .83 & 1.18 & .24\end{array}$

Note: All $p$ values for the correlations were below .001. As no predictions were made regarding the difference between the Fisher's r-to-z transformation, all $p$ values were two-tailed. 